\title{
The Effects of Sex and Sexual Orientation on Attractiveness Judgments: An Evolutionary Interpretation
}

\author{
William R. Jankowiak \\ Department of Anthropology, University of Nevada-Las Vegas, \\ Las Vegas, Nevada
}

\author{
Elizabeth M. Hill \\ Department of Psychiatry, University of Michigan, Ann Arbor, Michigan
}

\section{James M. Donovan \\ Department of Anthropology, Tulane University, New Orleans, Louisiana}

If attractiveness judgments reflect biologically important reproductive criteria, men should base judgments of potential partners on objective physical criteria more than do women; homosexuals and heterosexuals of the same sex should perceive attractiveness in the same terms, regardless of sex-object choice. To test this theory, photographs of men and women (20 each) were presented to members of four subject groups, solicited on an opportunistic basis. Subjects were asked to rank the sets of photographs separately on the dimensions of physical attractiveness and general social attractiveness. We found some sex differences across sexual orientation. There was less variation among men than women (heterosexual and homosexual) in evaluating the "good looks" of sex objects. Heterosexual and homosexual men ranked younger sex objects higher than older ones on "good looks." Heterosexual but not homosexual women ranked older sex objects higher. Sex had little effect on "social attractiveness" rankings, nor did putative age. These findings are interpreted as generally consistent with the existence of average sex differences in evaluative mechanisms that reflect different reproductive interests. Only further research, however, can identify the developmental origins of such differences.

\section{INTRODUCTION}

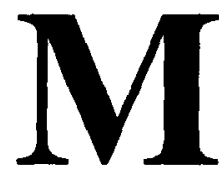

odern evolutionary theory posits that males and females will often use different behaviors to increase the representation of their genes in future generations (Hrdy and Williams 1983; Trivers 1972). Males of most species can increase reproductive

Received December 19, 1989; revised July 31, 1991

Address reprint requests to: William Jankowiak, Department of Anthropology, University of Nevada-Las Vegas, 4505 S. Maryland Parkway, Las Vegas, Nevada 89154. 
success by mating with several females, while females rarely benefit from multiple matings (Bateman 1948). In contrast, females more often increase reproductive success by increasing investment in offspring (Hrdy and Williams 1983; Trivers 1972). These sex differences in the routes to maximum fitness have been applied by sociobiologists and evolutionary psychologists to provide a basis for many aspects of human reproductive behavior (see reviews or articles in Chagnon and Irons 1979; Crawford et al. 1978: Daly and Wilson 1978; Symons 1979; Weinrich 1987).

Evolutionary considerations lead to the expectation that men and women will evaluate the opposite sex very differently. Men will discriminate and preferentially attempt to pair with women who are capable of reproducing. Thus, they will develop ways of discriminating reproductive value (Fisher 1930) and fertility of potential partners. Few specific physical and behavioral cues have been clearly demonstrated to reflect this quality, aside from those associated with age and health (Symons 1979; Thornhill and Thornhill 1983), but the argument has been made that concepts of female physical beauty correlate with precisely this reproductive quality (Buss 1987; Symons 1979; Williams 1975). The perceptual links between reproductive capability, cues to health (such as clear eyes or good muscle tone: Buss 1987), and judgments of attractiveness have not been fully clarified. To date. only sex differences in mate preferences for relative age have empirical support (Buss 1989; Kendrick and Keefe 1989).

In most ecological contexts, women will, on average, be less concerned with male youth, since it is less relevant to male fertility, than with their willingness and capability to invest in dependent offspring (Dickemann 1979; Trivers 1972). This quality could be signaled by personal characteristics like generosity, reliability, or reputation, in addition to acquired resources (reviewed by Ellis, in press; Hinde 1984). Few personal qualities have been empirically shown to indicate high mate value and high attractiveness specific to males, except for aspects of the good provider role (see Buss 1989). It does appear clear that in many cultures men more often report a matepreference for good-looking marriage partners, while women more often value male status or resources (Buss 1989; Buss and Barnes 1986: Townsend 1989; Townsend and Levy 1990). Cues that signal a male with high value as a potential mate are much less dependent on physique. Attractiveness ratings of variations in morphological features have failed to find a standard with high agreement (reviewed in Hill 1982).

Men and women may have developed psychological mechanisms that diverge in ways that promote reproductive interests (Alexander 1979; Cosmides and Tooby 1987; Symons 1979). Psychological mechanisms may modulate responses to potential romantic partners or competitors. Systems for sexual arousal, erotic attractions, and conscious evaluations of the opposite sex could all be influenced by biologically-based mechanisms. In studies in the U.S., manipulating displays of high status was associated with changes in perceived physical attractiveness as well as marital attractiveness (Hill 
et al. 1987; Townsend and Levy 1990). In this manner, other attractive features cloud reported perceptions of physical characteristics, acting like a reverse halo effect. The role and specificity of psychobiological mechanisms is currently a subject of much debate (Barkow 1989; Barkow, Cosmides, and Tooby, in press).

In their study of thousands of questionnaire respondents, Blumstein and Schwartz (1983) found that sex predicted the self-reported importance of one's partner's physical attractiveness, while there was little difference between homosexual and heterosexual people of the same sex. They concluded that physical beauty was less relevant to the happiness of lesbian couples. "While some lesbians respond to the dictates of fashion, many inhabit a culture scornful of what they consider male standards of female attractiveness, which they reject as indicators of women's worth"' (p. 250). Symons (1979) noted that differences by sex appear greater than by sexual orientation in the use of visual erotica, search for sexual variety, and duration of monogamous relationships. Based upon these empirical data, he argued for a biological as opposed to a cultural basis for sexual arousal mechanisms. He suggested that the similarities between heterosexual and homosexual men in evaluating sexual desirability by physical attractiveness and youth argued that these were "relatively 'innate' criteria" (p. 301). However, we know of no empirical reports of the effects of sexual orientation on attractiveness judgments.

This report attempts to expand and test Symons' ideas. We rely on men's and women's use of disparite criteria in judging attractiveness and propose that objective physical criteria will be used to a greater degree by men when called upon to judge women than by women judging men. We expect this disparity in criteria to be reflected in changes in the variance of judgments within a group (as used by Schulman and Hoskins 1986). We use homosexual men and women as a test case for the sex-specificity of attractiveness criteria. The assumption underlying this research strategy is that homosexuals differ from heterosexuals in object choice rather than other aspects of sexuality (cf. Symons 1979).

Even though reproductive strategies are very complex and modern environments are quite variable, we still predict detectible mean differences in how men and women assess another's attractiveness that reflect basic differences in reproductive strategies. In brief, we asked heterosexual and homosexual men and women to rank-order sets of photographs of men and women on two dimensions of attractiveness. One was physical, the other social. As ranking proceded, we solicited from these informants their descriptions of the basis for their judgments.

\section{Predictions}

(1) There will be less variation among men than women in evaluating the "good looks" of sex objects, whether they be men or women, since men's 
attractiveness judgments appear to be largely based on physical stimuli that are objectively salient.

(2) For men ranking sex objects, within-group consistency for "good looks" evaluations will be greater than for their "social attractiveness" evaluations, since men appear to use objective physical criteria for judging physical attractiveness, whereas social and personal compatibility is more idiosyncratic. Women's rankings should show the opposite relationship or show no difference by dimension.

(3) Men will rank younger sex objects higher than older ones on "good looks." Women will rank older stimuli somewhat higher or show little difference in ranks by age on "good looks," since, as discussed above, age is less relevant for women's than men's attractiveness judgments.

\section{METHODS}

\section{Stimulus Photographs}

Photographs were taken at random from a 1979 yearbook of a major private southern university. The selection process netted photographs of 35 females and 39 males, all Caucasian. Ten judges (five male, five female) were asked to rank each of the two photo sets on the criterion of "good looks." Using the averaged rank, the photographs were renumbered 1-35 (female) and 139 (male); if two photographs had an identical mean, the photo with the larger standard deviation was discarded (two males and two females were thus discarded). In order to obtain a manageable group of photographs representing a continuum of "good looks," the photographs were paired (e.g., $1-2,3-4$, etc.) and the member of a pair with the larger standard deviation was discarded. The last (unpaired) photo of each set was retained. This process resulted in a set of 17 females and 19 males; 2 additional males were discarded to establish numerical equality between the two sets.

Additionally, photographs of six males and seven females were taken from the directory of a graduate professional school. After being ranked and averaged according to the process described above, the top three photographs of each sex were selected for inclusion, adding an age range to the photo set. The final number in each set was twenty. High-quality photostatic copies were made so that identical photo sets could be provided to each of three interviewers. All responses are to copies of the sets; no subject viewed the originals.

\section{Participants}

Caucasian participants were solicited on an opportunistic basis by three interviewers. A total of 52 people were tested, 13 in each group (heterosexual men, homosexual men, heterosexual women, and homosexual women). All 
participants with Kinsey scores of 2-4 (the bisexual middle range) were excluded from analysis because we wanted to contrast clear groups in this preliminary investigation.

Although all interviewers attempted to solicit data from each of the four study populations, interviewers and subjects were not completely counterbalanced. Examination of data by interviewer indicates that this circumstance did not significantly bias the results. Homosexual participants were somewhat older than heterosexual. Since subject age could affect ranks for the older photograph subset, we tested whether the high variability in these ranks by the homosexual women could be due to an inadvertent correlation with age. We feel subject age is not a critical factor, however, since Spearman correlations are nonsignificant between subject age and average rank

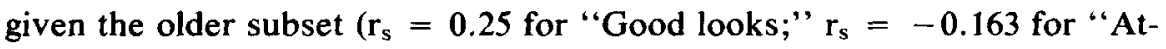
tractive').

\section{Procedure}

First, demographic information was obtained from the subject, including sex, age, and Kinsey score for sexual orientation. The subject was then asked to rank members of each set of photographs according to how "good-looking" and how "socially attractive" they were. "Good-looking" was preferred to "beautiful" because the latter frequently connotes feminine qualities whereas the former term is comparatively sex-irrelevant. If needed, clarification was offered that this evaluative judgment was to be based on physical appearance alone. If needed, clarification was offered that "Attractive" judgments need not be limited to physical criteria, but could be based on the following: "If you were at a party, with whom would you want to spend your time?" We thus refer to this quality as "social attractiveness." As ranking proceded, participants were asked to describe the criteria they were using. When rankings for a photograph greatly differed for the two dimensions, we probed for people's justifications for their judgments. These statements were recorded and provide some information about conscious rationales that may help interpretation of results. The order of presentation of male and female stimuli and the order of ranking dimension were alternated from one subject to the next.

\section{Statistical Analysis}

Consistency of judgments within groups was tested by calculating for each subject the absolute deviation of stimulus rank from the mean rank given that photograph by his or her group. These deviations were then averaged over the 20 photographs to obtain an average deviation for each subject. Since ranks were used, group differences in mean deviations were analyzed by Mann-Whitney tests. Main effects for differences between sexes and between preferences were tested by collapsing over the other factor. Inter- 
actions were tested by analyzing differences between the additive effect of one factor on the other factor (cf. Bradley 1968; pp. 138-141). When interactions were significant, we analyzed simple effects by testing a factor within one level of the other. Medians and interquartile ranges are presented. For the stimulus age comparison, mean ranks and mean deviations as calculated above were averaged over the younger photograph set $(n=17)$ and the older set $(\mathbf{n}=3)$. Deviations were analyzed as above. Group differences in mean ranks were also analyzed by Mann-Whitney tests.

\section{RESULTS}

\section{Consistency in Judgments of Good Looks and Attractiveness}

Figure 1 shows in the mean deviations for "good looks" rankings of sex objects. Sex objects are women for heterosexual men and homosexual women, and are men for heterosexual women and homosexual men. The mean deviation is the average absolute deviation from each group's mean. The deviation was significantly lower for men than for women $(\mathbf{U}=133$, $\mathbf{p}=0.000)$. Sexual orientation did not have a significant effect $(\mathbf{U}=258$, $\mathbf{p}=\mathbf{0 . 1 4 3}$ ). These results support our first prediction (sex differences in the variability of "good looks" judgments).

The mean deviation increased for everyone when shifting from evaluating "good looks" $(\mathbf{M}=3.11)$ to "social attractiveness" $(\mathbf{M}=3.53$; $\mathbf{U}=799, \mathbf{p}=0.000)$. Women $(\mathbf{M}=3.40)$ were more variable than men $(\mathbf{M}$

FIGURE 1. Within-group variability on "good looks" ranks; median and interquartile range are shown for absolute deviations from the group's mean rank on the "good looks" dimension (averaged over 20 photographs).

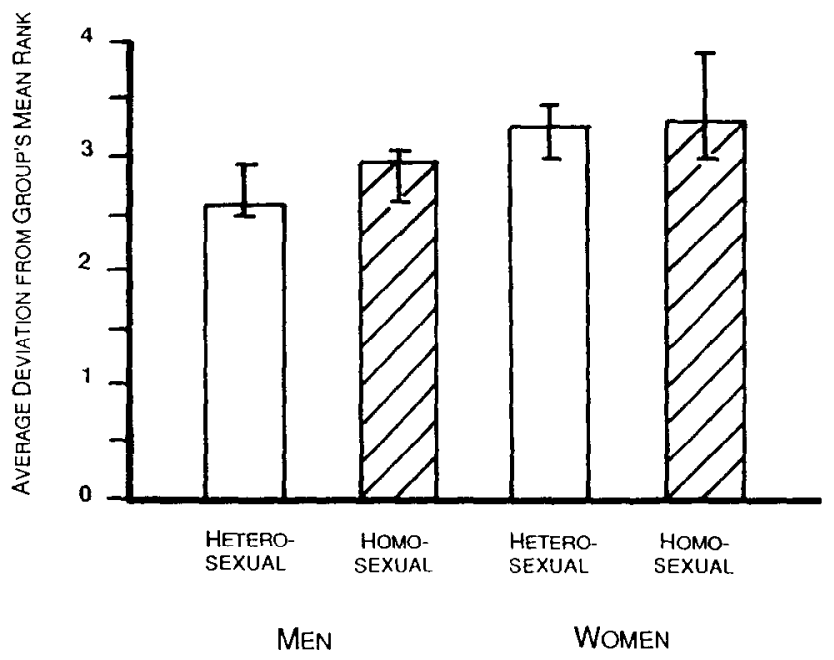




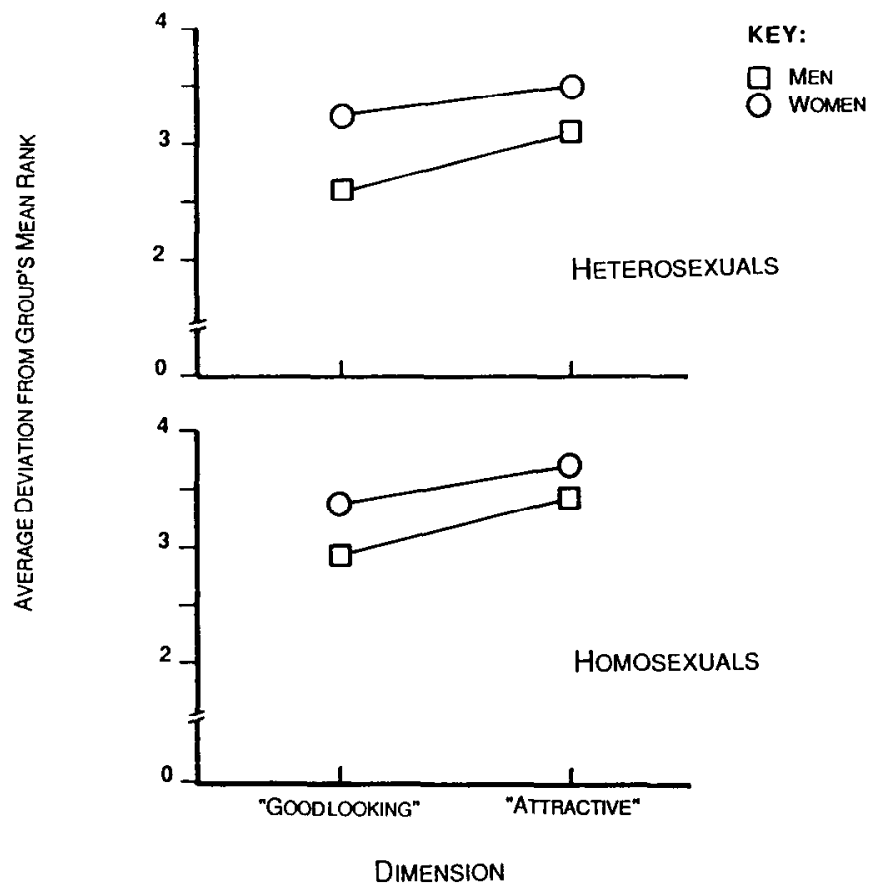

FIGURE 2. Effects of rater sex and ranking dimension on within-group variability (the average absolute deviation from the group's mean rank). The median is shown (averaged over the 20 photographs judged by each person).

$=3.24 ; \mathbf{U}=903, \mathbf{p}=0.003)$ and homosexuals $(\mathbf{M}=3.49)$ tended to be more variable than heterosexuals $(\mathbf{M}=3.15 ; \mathbf{U}=1083, \mathbf{p}=\mathbf{0 . 0 8 0})$. The change in variation by dimension was not as great for women as for men, however. Figure 2 shows the significant interaction between rater sex and ranking dimension on the mean deviations ( $\operatorname{sex} \times$ dimension, $U=198$, $\mathbf{p}=0.010$ ). The second prediction (males will show greater variance than females in ranking "social attractiveness" of sex objects) was supported in part: women were more variable overall; men showed a greater change in variance when shifting from good looks to social attractiveness ranks (Fig. 2).

Table 1 shows the within-group consistency of rankings for sex objects and non-sex objects. Overall, the consistency of men's rankings shifted more than women's in viewing sex objects versus others (sex $\times$ photo type, $\mathbf{U}=983, \mathbf{p}=0.016$ ). As predicted, this interaction was significant for "good looks" ranks (sex $\times$ photo type, $U=163, p=0.001$ ) but not for "social attractiveness" ( $\operatorname{sex} \times$ photo type, $U=322, \mathbf{p}=0.770$ ). Decomposing the interaction by testing it within each group separately, we found it statistically significant within the heterosexual group only ("good looks;" sex $\times$ photo type, $\mathrm{U}=25, \mathbf{p}=0.002$ ). 
Table 1. Consistency of Rankings Within Subject Groups

\begin{tabular}{|c|c|c|c|c|}
\hline \multirow{2}{*}{$\begin{array}{l}\text { Photograph } \\
\text { Type: } \\
\text { Ranking } \\
\text { Dimension: }\end{array}$} & \multicolumn{2}{|c|}{ Sex Objects } & \multicolumn{2}{|c|}{ Non-Sex Objects } \\
\hline & Good-looking & Attractive & Good-looking & Attractive \\
\hline \multicolumn{5}{|l|}{ Men } \\
\hline Heterosexual & 2.68 & 3.47 & 3.03 & 3.43 \\
\hline Homosexual & 2.90 & 3.43 & 3.31 & 3.64 \\
\hline Women & \multicolumn{2}{|c|}{$\bar{x}=3.12$} & \multicolumn{2}{|c|}{$\bar{x}=3.35$} \\
\hline Heterosexual & 3.22 & 3.49 & 2.75 & 3.09 \\
\hline Homosexual & 3.53 & 3.74 & 3.42 & 3.96 \\
\hline & \multicolumn{2}{|c|}{$\bar{x}=3.50$} & \multicolumn{2}{|c|}{$\bar{x}=3.31$} \\
\hline
\end{tabular}

Values shown are the average deviations (over 20 photographs) from the mean rank given by the group.

\section{The Interaction of Target Age with Rater Sex}

Ranks for younger stimuli $(\mathrm{n}=17)$ displayed less variation than for older stimuli $(\mathbf{n}=3)(\mathbf{M}=4.14$, older; 3.17, younger; $\mathbf{U}=7635, \mathbf{p}=0.000)$ for both "good looks" ( $\mathbf{M}=3.81$, older; 2.98 , younger; $\mathbf{U}=1984, \mathbf{p}=0.000)$ and "social attractiveness" $(\mathbf{M}=4.48$, older; 3.36 , younger; $\mathbf{U}=1851$, $\mathbf{p}=0.001$ ). The average ranks for the two subsets did not differ overall, however. There were no significant effects of age using the "social attractiveness" dimension alone. The average ranks for the photograph subsets did not differ consistently on "good looks" ranks because of an interaction with rater sex $(U=480, p=0.009)$.

As shown in box plots in Figure 3, this interaction was apparent only for the heterosexual group. They followed the predicted pattern for "good looks" judgments, with men giving higher ranks (less preferred) to older than to younger women, while women showed the opposite pattern (rater sex $\times$ stimulus age interaction; $U=139, \mathbf{p}=0.006$ ). The interaction was not significant for the homosexual group $(U=105, \mathbf{p}=0.293)$. For the homosexual group, the ranks given the older subset were significantly higher (less preferred) than those given the younger subset $(U=465, p=0.020)$. Our third prediction was basically supported by these results, with the exception of homosexual women.

\section{DISCUSSION}

Although many physical attractiveness studies have supported sex differences in preferences for beauty or status (reviewed in Buss 1989; Buss and Barnes 1986; Hill et al. 1987), few have used similar methods with heterosexual and homosexual participants. Our research examined whether the classification of someone as a sex object, whether male or female, affects rankings of photographs by men and women. We found that within-group 


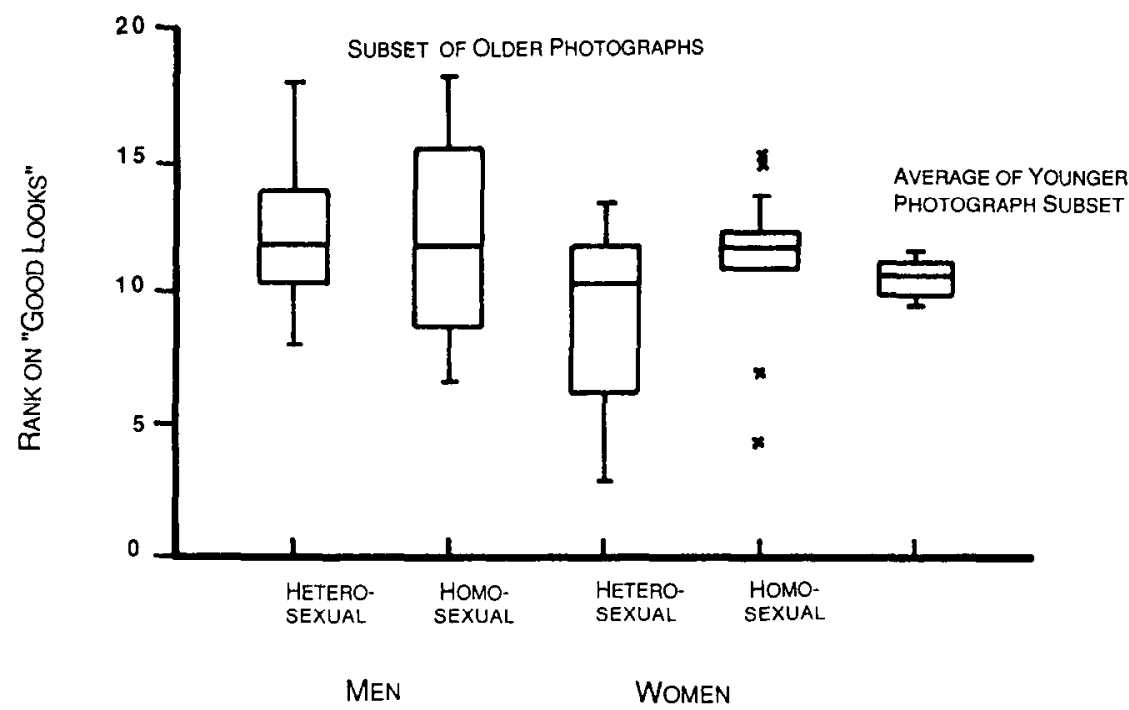

FIGURE 3. Effects of rater sex and relative age of photograph model, as shown by box plots of the average ranks given the older photograph subset $(n=3)$. The rank of the younger subset $(n=17)$ is shown for comparison. The median is denoted by a horizontal line. The interquartile range is boxed, and the entire range of the data is indicated by vertical lines. Other outliers are shown by asterisks.

consistency of rankings differed by sex, ranking dimension, age of target, and somewhat by sexual orientation.

Ranks were less variable on good looks, when men ranked sex objects, especially young ones, and when heterosexual women ranked other women. We feel that relative consistency or agreement in ranks within a group reflects the use of aspects of physical appearance. In summary, we found that (1) There was less variation among men than women in evaluating the "good looks" of sex objects, whether they were men or women. (2) Men and women both appeared to use idiosyncratic criteria when evaluating another's relative "social attractiveness." (3) Men ranked younger sex objects higher than older ones on "good looks." Heterosexual women ranked older men higher. Age of the target person also had little effect on social attractiveness.

Informants' justifications of their "good looks" rankings may illuminate the sex differences. Informants claimed that they were using objective criteria based on physical attributes. For instance, whenever men ranked sex objects they noted that "good looks" were marked by "wide eyes," "full hair," "youth," "nice complexion," and whether they were "sexually aroused and wanted to make love" to the person, male or female. Although women insisted they had ranked the subjects according to their relative "good looks," their reasons for their decisions usually invoked personality attributions and not physical descriptions. For example, women often noted that a "very good-looking" person was someone who looked "happy," 
"thoughtful," "smart and upbeat," or "fun to play with;" a person was considered not to be very good-looking if they looked "like losers," were "too young and inexperienced," "lifeless," or looked "too dangerous." This perceptual mixing may underlie most variability in "good-looking ranks, both for heterosexual women ranking men and homosexual women ranking women.

Others have described the intrusion of personality attributions into women's assessments of male physical attractiveness (Gregersen 1982; Remoff 1984). Weinrich (1987) employed the distinctions between "limerent" (Tennov 1979) and "lusty" sexual attractions and between impulses and responses. The term "limerent" attraction refers to the eroticization of a particular person's traits as a relationship progresses in intimacy. "Lusty" attractions are directed toward people who share characteristics with a specific idealized type (Weinrich 1987; p. 116). On average, there may be sex differences in the ease with which each type of response is elicited. Of relevance to the present study, when properties specific to an individual become attractive, there is no a priori physical standard by which people are judged.

However, both sexes can employ the other's typical criteria. When heterosexual women evaluated a woman's relative "good looks," they, unlike homosexual women, had no difficulty in justifying their selections with physical descriptions. For example, they noted that a good-looking woman was someone who had a "small nose," "nice hair," "wide eyes and full mouth." "pretty teeth," and so forth. On the other hand, personality factors seldom were mentioned by heterosexual men ranking women's physical attractiveness, although they were when these men ranked other men. Heterosexual men readily acknowledge, for example, that it was "easier" and "more fun" to rank women than men. In contrast, homosexual men expressed boredom with having to rank women and admitted that they preferred to rank a woman's "good looks" on the basis of assumed personality attributes. Homosexual men often justified this with, "looks are not as important as having a fun personality." Women considered "just pretty" or "cute" by heterosexuals, were deemed by male homosexuals not to be "very interesting" or "physically attractive" because they either were "too brainy" or "silly."

The impact of age of the photographed people on their relative "good looks" and "social attractiveness" differed by rater sex. We predicted and found that men would be less inclined than women to rank older sex objects as good-looking. However, age was not a factor when evaluating non-sex objects or in evaluating social attractiveness. Generally, rankings were more consistent for photographs of younger than for older pcoplc.

Heterosexual women judged photographs of older men to be better looking. One 21 year-old informant noted that "older men were better looking because you could talk to them about serious concerns; younger men were silly and not very serious about life and, in general, not very good-looking." For the homosexual group, older people were judged less attractive then 
younger on average (by both men and women raters). We think this resulted from the greater variability in rankings of the older subset, particularly by homosexual women. Some homosexual women thought older women were indeed "prettier;" whereas other informants did not. This clustering may reflect a mixture of criteria or of people who, for various reasons, tend toward more masculine or feminine criteria.

In justifying their ranks on "social attractiveness," a number of heterosexual and homosexual women commented that older sex objects were either "handsome" or "pretty" and were "turned on by them," but that the age discrepancy was not conducive to shared experiences and common interests. One 22 year-old female informant colorfully expressed her ambivalence over associating with an older man: "I am turned on by him, but he is too smart and out of my range." Men's evaluation of the social attractiveness of non-sex objects also reflected similar concerns with having common interests and values: even if an older person were "mature and handsome" he could be ranked lower in social attractiveness because they would not "have anything to talk about."

In summary, this study adds to our description of average sex differences in perception that are consistent with predictions from evolutionary theory. However, tests with adults who have experienced a homosexual subculture may not be conclusive if the subculture values mirror those in the wider, heterosexual realm. We think this unlikely, but cannot maintain that these sex differences in evaluating attractiveness are entirely "culture free." In any case, the developmental source of sex differences is unclear. Nevertheless, the fact that these differences are predictable from evolutionary theory, but not from any cultural theory of which we are aware, may indicate that the former is better for modeling the observed phenomena.

This manifestation of sex differences may partially result from the role of relationship type on criteria used in assessments, in particular, whether one is judging a partner for a short- or long-term relationship. The photograph-ranking task in the present experiment probably engaged short-term evaluative processes, exaggerating sex differences. Male criteria used in judgments of female attractiveness vary with duration or type of relationship; such changes are less apparent with women (Hill et al. 1987; Kendrick and Trost 1988; N. Thornhill 1989). Sex differences in preferences for long-term or marital partners are not as striking.

What needs evolutionary explanation may be sex differences in the tendency to be sensitive to context, rather than in a perceptual mechanism that attends to physique. Cosmides and Tooby (1987) contrast innate psychology to manifest psychology and behavior and discuss the problems of disentangling domain-specific mechanisms operating under different circumstances from more general mechanisms. Male standards of female "desirability" should be context-dependent ( $\mathrm{N}$. Thornhill 1989). In a specific case, it might be the optimal strategy for a man to find a stable partner for a long-term relationship; although there is less evidence for plasticity, women also should 
have facultative criteria for male attractiveness dependent upon factors like local sex differences in patterns of resource control (Hill et al. 1987; Smuts 1989). Future research can benefit by exploring such contextual factors in detail, along with more empirical research with homosexual participants.

We would like to acknowledge and thank Bob Anemone, Kathy Allen, Cliff Brown, Munro Edmonson, Kurt Hoop, Randolph Nesse, Don Symons, and John Townsend for their suggestions for improving the paper. Each co-author was responsible for writing the first draft of different sections of the paper. All of us are responsible for the final draft.

\section{REFERENCES}

Alexander, R. D. Darwinism and Human Affairs, Seattle, Washington: University of Washington Press, 1979.

Barkow, J. (Guest Ed.). Evolved constraints of cultural evolution. Special issue of Ethology and Sociobiology 10: Nos. 1-3, January, 1989.

Bateman, A. J. Intrasexual selection in Drosphila. Heredity 2: 349-368, 1948.

Cosmides, L., and Tooby, J. (Eds.). The Evolved Mind, Oxford: Oxford University Press, in press.

Blumstein, P., and Schwartz, P. American Couples, New York: Morrow, 1983.

Bradley, J. V. Distribution-free Statistical Tests, Englewood Cliffs, N.J.: Prentice Hall, 1968.

Buss, D. M. Sex Differences in human mate selection criteria: An evolutionary perspective. In Sociobiology and Psychology, C. Crawford et al. (Eds.). New York: Erlbaum, 1987.

- The evolution of human intrasexual competition: Tactics of mate attraction. Journal of Personality and Social Psychology 54: 616-628, 1988.

- Sex differences in human mate preferences: Evolutionary hypotheses tested in 37 cultures. Behavioral and Brain Sciences 12: 1-14, 1989.

- and Barnes, M. F. Preferences in human mate selection. Journal of Personality and Social Psychology 50: 559-570, 1986.

Chagnon, N. A., and Irons, W. G. (Eds.). Evolutionary Biology and Human Social Behavior: An Anthropological Perspective, North Scituate, MA: Duxbury, 1979.

Cosmides, L., and Tooby, J. From evolution to behavior: Evolutionary psychology as the missing link. In The Latest on the Best: Essays on Evolution and Optimality, J. Dupre (Ed.). Cambridge, MA: MIT, 1987.

Crawford, C., Smith, M., and Krebs, D. (Eds.). Sociobiology and Psychology: Ideas, Issues, and Applicutions, Hillsdale, NJ: Erlbaum, 1987.

Daly, M., and Wilson, M. Sex, Evolution and Behavior, North Scituate, MA: Duxbury, 1978.

Dickemann, M. The ecology of mating systems in hypergynous dowry societies. Social Sciences Information 18: 163-175, 1979.

Ellis, B. The evolution of sexual attraction: Evaluative mechanisms in men and women. In The Evolved Mind. J. Barkow, et al. (Eds.). Oxford: Oxford University Press, in press.

Fisher, R. A. The Genetical Theory of Natural Selection, Oxford: Clarendon Press, 1930.

Gregersen, E. Sexual Practices: The Story of Human Sexuality, New York: Watts, 1982.

Hill, E. M. Ornamentation and physical attractiveness: The influence of physique and status displays on physical, marital, and sexual attractiveness, Ph.D. Dissertation, Tulane University, 1982.

- Nocks, E. S., and Gardner, L. Physical attractiveness: Manipulation by physique and status displays. Ethology and Sociobiology 8: 143-154, 1987.

Hinde, R. A. Why do the sexes behave differently in close relationships? Journal of Social and Personal Relations 1: 471-501, 1984.

Hrdy, S. B., and Williams, G. C. Behavioral biology and the double standard. In Social Behavior of Female Vertebrates. S. K. Wasser (Ed.). New York: Academic Press, 1983. 
Kenrick, D. T., and Keefe, R. C. Time to integrate sociobiology and social psychology. Behavioral and Brain Sciences 12: 24-26, 1989.

- and Trost, M. R. A reproductive exchange model of heterosexual relationships: Putting proximate economics in ultimate perspective. In Review of Personality and Social Psychology, Vol. 10, C. Hendrick (Ed.). New York: Sage, 1988.

Remoff, H. T. Sexual Choice, New York: Dutton/Lewis, 1984.

Schulman, G. I., and Hoskins, M. Perceiving the male versus the female face. Psychology of Women Quarterly 10: 141-154, 1986.

Smuts, R. W. Behavior depends on context. Behavioral and Brain Sciences 12: 33-34, 1989.

Symons, D. The Evolution of Human Sexuality, New York: Oxford University Press, 1979.

Tennov, D. Love and Limerence-The Experience of Being in Love, New York: Stein and Day, 1979.

Thornhill, N. W. Characteristics of female desirability: Facultative standards of beauty. $B e$ havioral and Brain Sciences 12: 35-36, 1989.

Thornhill, R., and Thornhill, N. W. Human rape: An evolutionary analysis. Ethology and Sociobiology 4: 63-99, 1983.

Townsend, J. M. Mate-selection criteria: A pilot study. Ethology and Sociobiology 10: $241-253,1989$.

- , and Levy, G. D. Effects of potential partners' physical attractiveness and socioeconomic status on sexuality and partner selection: Sex differences in reported preferences of university students. Archives of Sexual Behavior 19: 149-164, 1990.

Trivers, R. L. Parental investment and sexual selection. In Sexual Selection and the Descent of Man, B. Campbell (Ed.). New York: Aldine, 1972.

Weinrich, J. D. Sexual Landscapes: Why We Are What We Are/Why We Love Whom We Love, New York: Scribners, 1987.

Williams, G. C. Sex and Evolution, Princeton, NJ: Princeton University Press, 1975. 